\title{
The Phylogeny of Methanopyrus kandleri
}

\author{
MARIA C. RIVERA* AND JAMES A. LAKE \\ Molecular Biology Institute and Department of Biology, University of California, Los Angeles, \\ Los Angeles, Califormia 90024
}

\begin{abstract}
The phylogenetic position of Methanopyrus kandleri has been difficult to determine because reconstructions of phylogenetic trees based on rRNA sequences have been ambiguous. The most probable trees determined by most algorithms place the genus Methanopyrus at the base of a group that includes the halobacteria and the methanogens and their relatives, although occasionally some algorithms place this genus near the eocytes (the hyperthermophilic, sulfur-metabolizing prokaryotes), suggesting that it may belong to this lineage. In order to resolve the phylogeny of the genus Methanopyrus, we determined the sequence of an informative region of elongation factor 1-alpha that contains an 11-amino-acid insertion in eocytes and eukaryotes which is replaced by a 4-amino-acid insertion in methanogens, halobacteria, and eubacteria. On the basis of the results of our elongation factor 1-alpha gene analysis, we concluded that the genus Methanopyrus diverged from the eocyte branch before the eukaryotic and eocyte lineages separated and therefore is not an eocyte.
\end{abstract}

Methanopyrus kandleri is a rod-shaped, gram-positive, methanogenic bacterium that was isolated from a deep thermal vent in the Gulf of California (16). This organism is the first known hyperthermophilic methanogen that grows at temperatures up to $110^{\circ} \mathrm{C}(16)$. Although extreme thermophily has been found in three phylogenetically different groups of prokaryotes (the eubacteria, the methanogens and their relatives, and the eocytes [hyperthermophilic, principally sulfur-metabolizing prokaryotes]), members of the genus Pyrodictium are the only other prokaryotes that are known to grow at a temperature of $110^{\circ} \mathrm{C}$ or above $(37,38)$. On the basis of the results of a $16 \mathrm{~S}$ rRNA and protein synthesis elongation factor 1-alpha (EF-1 $\alpha$ ) sequence analysis, the genus Pyrodictium was classified as an eocyte taxon $(24,32,33,37)$; therefore, we wanted to determine whether the genus Methanopyrus is also an eocyte taxon.

Methanopyrus kandleri appears to represent a unique lineage within the phylogenetically diverse methanogens $(18,30)$, and, consistent with this, the results of a Jukes-Cantor distance and parsimony analysis of the 16S rRNA sequence of this organism have shown that it is only distantly related to the other methanogens (8). A suggestion that $M$. kandleri may be related to the eukaryotes resulted from the discovery that a eukaryoticlike topoisomerase I molecule is present in Methanopyrus cells (35).

The most probable tree determined in our analysis of smallsubunit rRNA sequences in which the Jukes-Cantor (19), Kimura (21) and paralinear distance (27) algorithms were used is shown in Fig. 1; the probabilities of this eocyte tree are 72.5, 60.5 , and $63.5 \%$, respectively, and the probabilities of the archaebacterial tree (data not shown) are 20.5, 29.5, and $20.0 \%$, respectively. The most probable location of the genus Methanopyrus on the trees obtained with these three algorithms is at the branch labelled A; branch B is less likely, and branch C is least likely. The trees that have been constructed, the similarity of the maximal growth temperatures of Methanopyrus and Pyrodictium strains, and the presence of a eukaryotic topoisomerase I-like molecule all raised the possibility that the genus Methanopyrus may be more closely related to eukaryotes than previously thought. Given that sequence analysis studies can be biased by unequal rate effects, by site-to-site variation, and by the order of alignment, we sought additional, more reliable

* Corresponding author. evidence for the phylogenetic position of the genus Methanopyrus.

In a previous study, the phyletic distribution of the two variants of EF-Tu and EF- $1 \alpha$ was used to infer an immediate relationship between the eocytes and the eukaryotes (32). Previously, this relationship had been difficult to analyze by direct sequence comparisons because of the extremely long divergence times between the prokaryotes and the eukaryotes. In that study, we showed that the members of the genus Pyrodictium and the other eocytes, as well as all of the eukaryotes, contain an 11-amino-acid segment in EF- $1 \alpha$ that is not present in other prokaryotes. In the methanogens, the halobacteria, and the eubacteria this segment is replaced by a 4-amino-acid segment. In light of the questionable phylogenetic position of the genus Methanopyrus, determining the sequence of the EF- $1 \alpha$ gene of a member of this taxon could reveal if it is also an immediate eukaryotic relative (containing the 11-aminoacid segment and corresponding to tree branch C) or if it is more closely related to the methanogens and halobacteria (containing the 4-amino-acid segment and corresponding to either tree branch $\mathrm{A}$ or tree branch $\mathrm{B}$ ).

As previously noted $(22,28,31)$, EF- $1 \alpha$ appears to be one of the best molecules available for studying deep divergences. This molecule is a slowly evolving protein found in all cells, where it binds, transports, and participates in the selection of the correct aminoacyl-tRNA. In addition, EF-1 $\alpha$ interacts with cellular components encoded by genes dispersed throughout the genome, including aminoacyl-tRNAs, ribosomal proteins, $16 \mathrm{~S}$ and $18 \mathrm{~S}$ rRNAs, and elongation factor EF-Ts; therefore, it is unlikely that EF-1 $\alpha$ is transferred laterally between organisms.

To determine whether the genus Methanopyrus is an eocyte genus, we isolated by PCR, cloned, sequenced, and analyzed a fragment in the GDP-binding domain of the gene coding for EF-1 $\alpha$ from several taxa whose sequences had not been determined previously. Total genomic DNAs were isolated from frozen cell pastes of Methanopyrus kandleri, Archaeoglobus fulgidus (36), and Thermodiscus maritimus by using the sodium dodecyl sulfate-proteinase K lysis method (14). Total nucleic acids were extracted from Dactylococcopsis salina (40) and purified by $\mathrm{CsCl}$ centrifugation (1). Degenerate oligonucleotide primers were designed on the basis of conserved amino acid motifs in all known EF-Tu and EF-1 $\alpha$ sequences (Fig. 2) and used for PCR amplification (34). The primers spanned the 


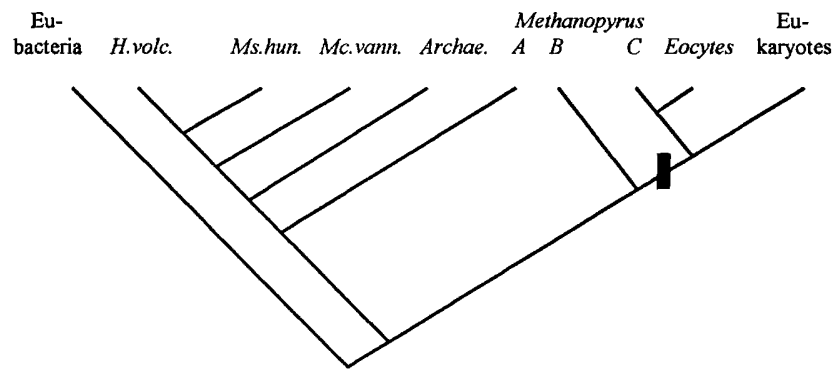

FIG. 1. Rooted tree showing the three possible positions of the Methanopyru. lineage. This tree is the most probable tree derived from an analysis of $16 \mathrm{~S}$ and $18 \mathrm{~S}$ rRNA sequences in which the Kimura (21), Jukes-Cantor (19), and paralinear distance $(25,27)$ algorithms were used. The tree is rooted in the branch leading to the eubacteria, as proposed by other workers $(12,17)$. The three possible locations of the genus Methanopyrus that have finite probabilities are designated branches $\mathrm{A}, \mathrm{B}$, and $\mathrm{C}$. The eubacterium used was Thermotoga maritima. Abbreviations: H.volc., Halobacterium volcanii; Ms.hun., Methanospirillum hungatei; Mc.vann., Methanococcus vannielii; Archae., Archaeoglobus fulgidus.

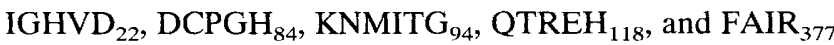
regions of the homologous Escherichia coli amino acid sequence (the subscripts indicate the positions of the $5^{\prime}$-most nucleotides of the primers in the E. coli EF-Tu sequence [9, 20]). The PCR fragments were cloned into the pT7 Blue vector (Novagen). The sequences of clones containing the PCR insertion were determined by using a Sequenase 2.0 kit (U.S.
Biochemicals) and M13 universal and reverse primers for reading both strands of the amplified fragments.

The nucleotide sequences of the methanogen Methanopyrus kandleri, Archaeoglobus fulgidus, the halophilic cyanobacterium Dactylococcopsis salina, and the eocyte Thermodiscus maritimus are shown in Fig. 3. The deduced amino acid sequence of the Methanopyrus kandleri EF- $1 \alpha$ fragment was aligned with the sequences of the homologous fragments of eocytes, methanogens and their relatives, a halobacterium, eubacteria, and eukaryotes (Fig. 2). The Methanopyrus kandleri sequence contained the 4-amino-acid segment (GVMP) characteristic of eubacteria, halobacteria, and methanogens, and it lacked the 11-amino-acid segment found in eocyte and eukaryotic sequences (Fig. 2).

The absence of the 11-amino-acid segment in the genus Methanopyrus is most parsimoniously consistent with branches $\mathrm{A}$ and $\mathrm{B}$ on Fig. 1. On either branch A or branch B, only a single change from the 4-amino-acid segment to the 11-aminoacid segment (Fig. 1, solid rectangle) is needed to explain the observed distribution of insertions. In comparison, one additional change from the 11-amino-acid segment to the 4-aminoacid segment on branch $\mathrm{C}$ would be required to explain the branch $\mathrm{C}$ alternative. Therefore, these results strongly indicate that the genus Methanopyrus is not an eocyte genus and is less closely related to the eukaryotes. In addition, it should be noted that all of the new sequences described in this paper are consistent with and provide additional support for the eocyte tree, on which the eocytes are the closest relatives of the eukaryotes.

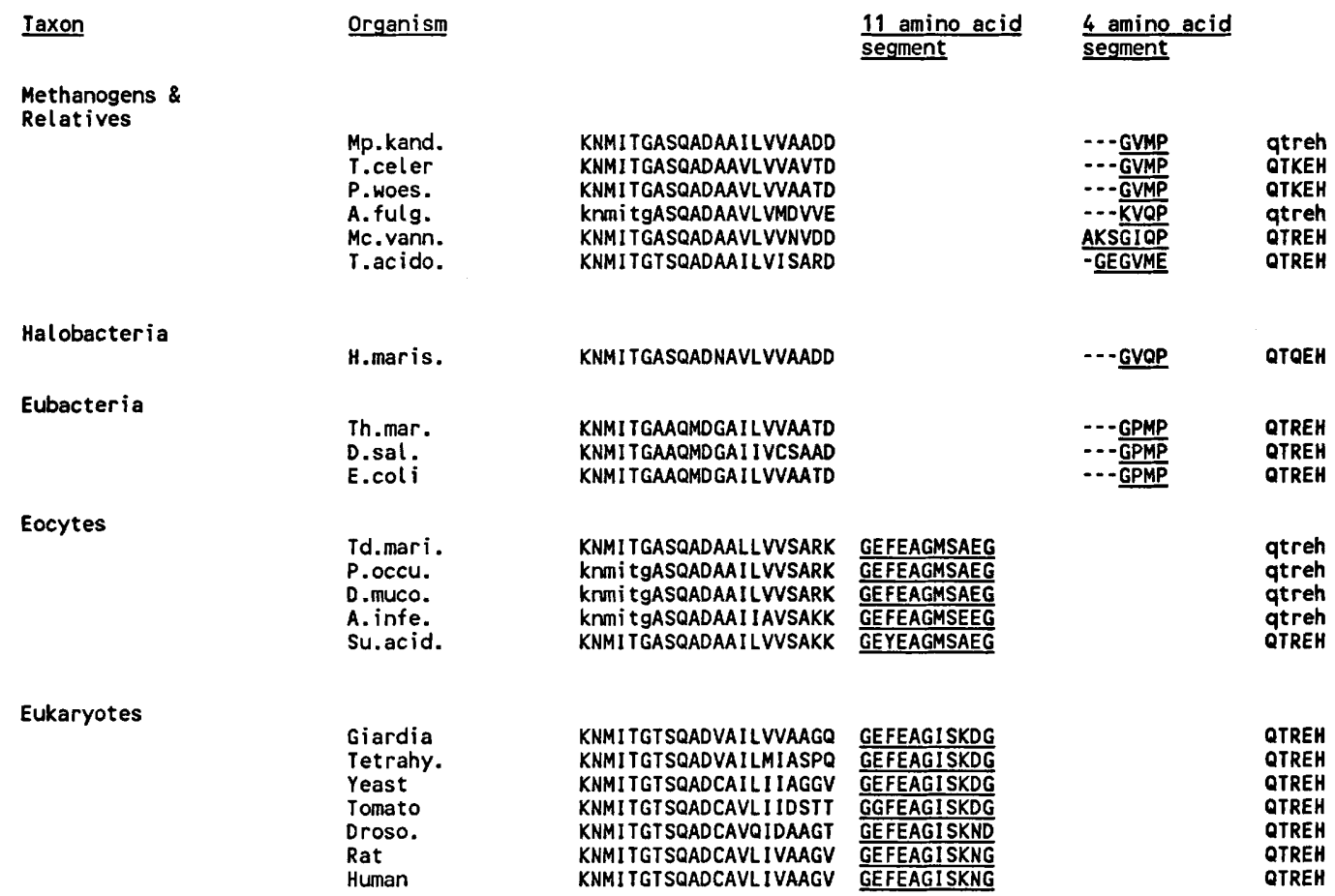

FIG. 2. Comparison of the Methanopyrus kandleri EF-1 $\alpha$ sequence with the sequences of methanogens, halobacteria, eubacteria, eocytes, and eukaryotes. The 4and 11-amino-acid segments are underlined. The lowercase letters represent sequences used as the PCR primers. The methanogens and their relatives which we used were Methanopyrus kandleri (Mp.kand.) (this study), Thermococcus celer (T.celer) (3), Pyrococcus woesei (P.woes.) (11), Archaeoglobus fulgidus (A.fulg.) (this study), Methanococcus vannielii (Mc.vann.) (26), and Thermoplasma acidophilum (T.acido.) (39). The halobacterium used was Halobacterium marismortui (H.maris.) (6). The eubacterial used were the thermophilic eubacterium Thermotoga maritima (Th.mar.) (5), the halophilic cyanobacterium Dactylococcopsis salina (D.sal.) (this study), and the enterobacterium E coli (9). The eocytes used were Thermodiscus maritimus (Td.mari.) (this study), Pyrodictium occultum (P.occu.) (32), Desulfurococcus mucosus (D.muco.) (32), Acidianus infermus (A.infe.) (32), and Sulfolobus acidocaldarius (Su.acid.) (4). The eukaryotes used were Giardia lamblia (Giardia) (13), Tetrahymena pyriformis (Tetrahy.) (23), Saccharomyces cerevisiae (Yeast) (10), Lycopersicon esculentum (Tomato) (29), Drosophila melanogaster (Droso.) (15), Rattus nonegicus (Rat) (2), and Homo sapiens (Human) (7). 


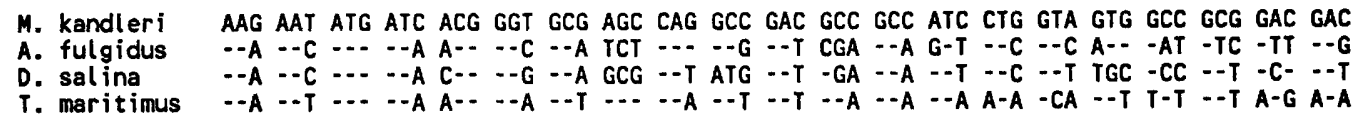

\section{M. kandleri \\ A. fulgidus \\ D. salina \\ T. maritimus}

GGA GAA TIT GAA GCA GGA ATG AGC GAA GAA GGT

GGT GTG ATG CCG CAG ACC AGA GAA CAC

AAG GTG CA- - - G $\cdots$ -

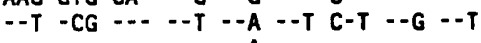

FIG. 3. Nucleotide sequences of the $\mathrm{KNMITG}_{95}-\mathrm{QTREH}_{118}$ fragments from Methanopyrus kandleri, Archaeoglobus fulgidus, Dactylococcopsis salina, and Thermodiscus maritimus. Triplets corresponding to amino acids are separated by spaces. Gaps are represented by solid lines. Dashes represent nucleotides identical to the ones in the $M$. kandleri reference sequence (top row).

Support for the hypothesis that 11-amino-acid segments occur in eukaryotes and eocytes and 4-amino-acid segments or their variants occur in all other prokaryotes was provided by the new sequences described in this paper. These sequences include the sequences of the extremely halophilic eubacterium Dactylococopsis salina, the sulfate reducer Archaeoglobus fulgidus, the hyperthermophilic methanogen Methanopyrus kandleri, and the eocyte Thermodiscus maritimus. Furthermore, the fact that this pattern continues to be found in new sequences from taxa that occupy other critical phylogenetic positions, such as Giardia lamblia (13), provides additional evidence that the EF-1 $\alpha$ segment is useful as a phylogenetic marker. We concluded that the genus Methanopyrus is either a member of the true archaebacteria (corresponding to branch A) or a member of the nonarchaebacterial, paraphyletic clade of organisms (including organisms like Thermococcus celer and Pyrococcus woesei) which are located before the node which gives rise to the eocytes and eukaryotes (branch B).

We thank Karl Stetter for providing Methanopyrus, Archaeoglobus, and Thermodiscus cells and thank Anthony Walsby for providing Dactylococcopsis cells.

This work was supported by a grant from the National Science Foundation to J.A.L.

\section{REFERENCES}

1. Anderson, L. K., and F. A. Eiserling. 1985. Plasmids of the cyanobacterium Synechocystis 6701. FEMS Microbiol. Lett. 29:193-195.

2. Ann, D. K., I. K. Moutsatsos, T. Nakamura, H. H. Lin, P. L. Mao, M. J. Lee, S. Chin, R. K. H. Liem, and E. Wang. 1991. Isolation and characterization of the rat chromosomal gene for a polypeptide ( $\mathrm{pS} 1$ ) antigenically related to statin. J. Biol. Chem. 266:10429-10437.

3. Auer, J., G. Spicker, and A. Bock. 1990. Nucleotide sequence of the gene for elongation factor EF-1 from the extreme thermophilic archaebacterium Thermococcus celer. Nucleic Acids Res. 18:3989.

4. Auer, J., G. Spicker, L. Meyerhofer, G. Puhler, and A. Bock. 1991. Organization and nucleotide sequence of the gene cluster comprising the elongation factor $1 \alpha$ from Sulfolobus acidocaldarius. Syst. Appl. Microbiol. 14:14-20.

5. Bachleitner, M., W. Ludwig, K. O. Stetter, and K. H. Schleifer. 1989. Nucleotide sequence of the gene coding for elongation factor $\mathrm{Tu}$ from the extreme thermophilic eubacterium Thermotoga maritima. FEMS Microbiol. Lett. 57:115-120.

6. Baldacci, G., F. Guinet, J. Tillit, G. Zaccai, and A. M. de Recondo. 1990 Functional implications related to the gene structure of the elongation facto EF-Tu from Halobacterium marismortui. Nucleic Acids Res. 18:507-511.

7. Brands, J. H., J. A. Maassen, F. J. van Hemert, R. Amons, and W. Moller. 1986. The primary structure of the subunit of human elongation factor 1 : structural aspects of guanine-binding nucleotide-binding sites. Eur. J. Biochem, 155:167-171.

8. Burggraf, S., K. O. Stetter, P. Rouviere, and C. R. Woese. 1991. Methanopyrus kandleri: an archeal methanogen unrelated to all other known methanogens. Syst. Appl. Microbiol. 14:346-351.

9. Clark, B. F. C., M. Kjeldgard, T. F. M. la Cour, S. Thirup, and J. Nyborg. 1990. Structural determination of the functional sites of $E$. coli elongation factor Tu. Biochim. Biophys. Acta 1050:203-208.

10. Cottrelle, P., D. Thiele, V. L. Price, S. Memet, J. Y. Micoin, C. Marck, J. M. Buhler, A. Sentenac, and P. Fromageot. 1985. Cloning, nucleotide sequence, and expression of one of two genes for yeast elongation factor 1 alpha. $\mathbf{J}$. Biol. Chem. 260:3090-3096.

11. Creti, R., F. Citarella, O. Toboni, A. M. Sanangelantoni, P. Palm, and P. Cammarano. 1991. Nucleotide sequence of a DNA region comprising the gene for elongation factor 1 from the ultrathermophilic archaeote Pyrococcus woesei, phylogenetic implications. J. Mol. Evol. 33:332-342.

12. Gogarten, J. P., H. Kibak, P. Dittrich, L. Taiz, B. J. Bowman, M. F. Manolson, R. J. Poole, T. Date, T. Oshima, et al. 1989. Evolution of the vacuolar H+-ATPase: implications for the origin of eukaryotes. Proc. Natl. Acad. Sci. USA 86:6661-6665.

13. Hashimoto, T., Y. Nakamura, F. Nakamura, T. Shirakura, J. Adachi, N. Goto, K. Okamoto, and M. Hasegawa. 1994. Protein phylogeny gives a robust estimation for early divergences of eukaryotes: phylogenetic place of a mitochondria-lacking protozoan, Giardia lamblia. Mol. Biol. Evol. 11:65-71.

14. Herrmann, B. G., and A. Frischauf. 1987. Isolation of genomic DNA. Methods Enzymol. 152:180-183.

15. Hovemann, B., S. Richter, U. Walldorf, and C. Cziepluch. 1988. Two genes encode related cytoplasmic elongation factors 1 alpha (EF-1 alpha) in Drosophila melanogaster with continuous and stage specific expression. Nucleic Acids Res. 16:3175-3194.

16. Huber, R., M. Kurr, H. W. Jannasch, and K. O. Stetter. 1989. A novel group of abyssal methanogenic archaebacteria (Methanopynus) growing at $110^{\circ} \mathrm{C}$. Nature (London) 342:833-834.

17. Iwabe, N., K. Kuma, M. Hasegawa, S. Osawa, and T. Miyata. 1989. Evolutionary relationship of archaebacteria, eubacteria, and eukaryotes inferred from phylogenetic trees of duplicated genes. Proc. Natl. Acad. Sci. USA 86:9355-9359.

18. Jones, J. J., D. P. Nagle, and W. B. Whitman. 1987. Methanogens and the diversity of archaebacteria. Microbiol. Rev. 51:135-177.

19. Jukes, T. H., and C. R. Cantor. 1969. Evolution of protein molecules, p. 21-132. In H. N. Munro (ed.), Mammalian protein metabolism. Academic Press, New York.

20. Jurnak, F., S. Heffron, B. Schick, and K. Delaria. 1990. Three-dimensional models of the GDP and GTP forms of the guanine nucleotide domain of Escherichia coli elongation factor Tu. Biochim. Biophys. Acta 1050:209-214.

21. Kimura, M. 1983. The natural theory. Cambridge University Press, London.

22. Kraus, M., M. Gotz, and W. Loffelhardt. 1990. The cyanelle str operon from Cyanophora paradoxa: sequence analysis and phylogenetic implications. Plant Mol. Biol. 15:561-565.

23. Kurasawa, Y, O. Numata, M. Katoh, H. Hirano, J. Chiba, and Y. Watanabe. 1992. Identification of Tetrahymena 14-nm filament-associated protein as elongation factor 1 alpha. Exp. Cell Res. 203:251-258.

24. Lake, J. A. 1988. Origin of the eukaryotic nucleus determined by rateinvariant analysis of rRNA sequences. Nature (London) 331:184-186

25. Lake, J. A. 1994. Reconstructing evolutionary trees from DNA and protein sequences: paralinear distances. Proc. Natl. Acad. Sci. USA 91:1455-1459.

26. Lechner, K., and A. Bock. 1987. Cloning and nucleotide sequence of the gene for an archaebacterial protein synthesis elongation factor Tu. Mol. Gen. Genet. 208:523-528.

27. Lockhart, P. J., M. A. Steel, M. D. Hendy, and D. Penny. 1994. Recovering evolutionary trees under a more realistic model of sequence evolution. Mol. Biol. Evol. 11:605-612.

28. Moldave, K. 1985. Eukaryotic protein synthesis. Annu. Rev. Biochem. 54: 1109-1125.

29. Pokalsky, A. R., W. R. Hiatt, N. Ridge, R. Rasmussen, et al. 1989. Structure and expression of elongation factor-1-alpha in tomato. Nucleic Acids Res. 17:4661-4673.

30. Reeve, J. N. 1992. Molecular biology of methanogens. Annu. Rev. Microbiol. 46:165-191.

31. Riis, B., S. I. S. Rattan, B. F. C. Clark, and W. C. Merrick. 1990. Eukaryotic protein elongation factors. Trends Biol. Sci. 15:420-423.

32. Rivera, M. C., and J. A. Lake. 1992. Evidence that eukaryotes and eocyte prokaryotes are immediate relatives. Science 257:74-76.

33. Runnegar, B. 1993. Proterozoic eukaryotes: evidence from biology and ge- 
ology, p. 287-297. In S. Bengtson (ed.), Early life on earth. Cambridge University Press, Cambridge.

34. Saiki, R. K., D. H. Gelfand, S. Stoffer, S. J. Scharf, R. Higuchi, G. T. Horn, K. B. Mullis, and H. A. Erlich. 1988. Primer-directed enzymatic amplification of DNA with a thermostable DNA polymerase. Science 239:487-491.

35. Slesarev, A. I., K. O. Stetter, J. A. Lake, M. Gellert, R. Krah, and S. A. Kozyavkin, 1993. DNA topoisomerase $\mathrm{V}$ is a relative of eukaryotic topoisomerase I from a hyperthermophilic prokaryote. Nature (London) 364:735737.

36. Stetter, K. O. 1988. Archaeoglobus fulgidus gen. nov., sp. nov.: a new taxon of extremely thermophilic archaebacteria. Syst. Appl. Microbiol. 10:172-173.
37. Stetter, K. O., G. Fiala, G. Huber, R. Huber, and A. Segerer. 1990. Hyperthermophilic microorganisms. FEMS Microbiol. Rev. 75:117-124.

38. Stetter, K. O., H. Konig, and E. Stackebrandt. 1983. Pyrodictium gen. nov., a new genus of submarine disc-shaped sulfur reducing archaebacteria growing optimally at $105^{\circ} \mathrm{C}$. Syst. Appl. Microbiol. 4:535-551.

39. Tesch, A., and F. Klink. 1990. Cloning and sequencing of the gene coding for the elongation factor 1-alpha from the archaebacterium Thermoplasma acidophilum. FEMS Microbiol. Lett. 71:293-297.

40. Walsby, A. E., J. Van Rijn, and Y. Cohen. 1983. The biology of new gasvacuolate cyanobacterium, Dactylococcopsis salina sp. nov., in Solar Lake. Proc. R. Soc. Lond. B Biol. Sci. 217:417-447. 